\title{
Multisource Evidence for Final State Hadrons in 3.7A GeV ${ }^{16} \mathrm{O}-$ Nucleus Interactions
}

\author{
W. Osman, M. Fayed, and Mennatallah. M. M \\ Physics Department, Faculty of Science, Cairo University, Giza, Egypt. \\ Email: menna_mhm8682@yahoo.com
}

\begin{abstract}
In $3.7 \mathrm{~A} \mathrm{GeV}{ }^{16} \mathrm{O}$ interactions with emulsion nuclei, the inelastic interaction cross section of ${ }^{16} \mathrm{O}$ in nuclear emulsion is approximated as a function of the target mass number. In the present energy region, the cross section is independent on the energy. It can be determined in the light of the Glauber's multiple scattering theory.

The multiplicity characteristics of shower particles produced in $3.7 \mathrm{~A} \mathrm{GeV}{ }^{16} \mathrm{O}$ interactions with emulsion nuclei are studied. The effect of the projectile and target sizes on the shower particles production is examined. The produced shower particles are discriminated into forward ones that produced in the forward angular zone (FHS) and the backward ones that produced in the backward angular zone (BHS). The interaction probability with each target nuclei $(\mathrm{H}, \mathrm{CNO}, \mathrm{Em}, \mathrm{and} \mathrm{AgBr})$ is simulated on the basis of Glauber's multiple scattering theory approaches. The forward emitted shower particles multiplicity distributions are usually characterized by Poisson peaking shapes, which imply multisource superposition These sources depend on the system size, centrality, and incident energy. Therefore these particles can originate from fireball nuclear matter or hadronic nuclear matter. The backward emitted shower particles multiplicity distributions are usually characterized by decay shape. However, this shape can deform to shoulder shape at higher centralities. The multiplicity characteristics depend only on the target size regarding the limiting fragmentation hyposises at $\mathrm{E}_{\mathrm{lab}} \sim 3-4 \mathrm{GeV}$ and projectile mass number $\geq 6$. The results indicate that a single target source is mainly responsible for shower particles creations. Particle production and other secondary source can be contributed at higher centralities. The data are simulated in the framework of the modified FRITIOF model. The multisource thermal model can predict source numbers responsible for particle production. The modified FRITIOF model can predict the system of the relativistic hadron production in the BHS well. In the FHS the hadronization system can be described satisfactorily. The decay shape indicates a single source production system and the peaking one is a multisource superposition. This is confirmed well by the multisource thermal model predictions.
\end{abstract}

\section{Key Words}

${ }^{16}$ O Interactions/ Dubna Energy/ Hadron Sources/ Modified FRITIOF Model/ Multi Source Thermal Model.

\section{PACS}

25.75.-q, 25.75.Dw, 25.75.Gz, 25.75.Ld, 25.70.Mn, 25.70.Pq, 41.75.Ak, 41.75.Cn, 29.40.Rg, 07.68.+m

\section{Academic Discipline and Sub-Disciplines}

Physics - High energy physics.

\section{SUBJECT CLASSIFICATION}

\author{
High energy physics - Particle physics.
}

\section{TYPE (METHOD/APPROACH)}

In photographic nuclear emulsion nomenclature the shower particles are those flying with the relativistic velocity, the so-called relativistic hadrons. They are separated from the non-interacting singly charged projectile fragments (protons, deuterons, and tritons) and differentiated from them. The charged pions are regarded as the major fraction part of the produced shower particles (more than 90\%). The neutral pions cannot be observed directly in the photographic nuclear emulsion. The average number of the neutral pions produced from any interaction is determined by the half of the average number of the charged pions or by using heavy ion interactions at Dubna energy. Hence any associated trend observed for the charged pions can be regarded by the neutral ones. In this experiment the produced pions are classified according to the emission zone in the $4 \pi$ space, into two groups. One of both encloses those emitted in the forward hemisphere, FHS, at $\theta_{\mathrm{lab}}<90^{\circ}$. The other encloses those emitted in the backward hemisphere, BHS, at $\theta_{\mathrm{lab}} \geq 90^{\circ}$. The used Dubna energy, 3.7A GeV, can be considered the onset of the nuclear limiting fragmentation, NLF, $\left(E_{l a b} \geq 1 \mathrm{~A} \mathrm{GeV}\right)$. Nuclear emulsion usage can give a wide range of target nuclear size $\left(A_{T}=1\right.$ up to 108). Explained briefly in the FRITIOF model is the particle production mechanism at relativistic and ultra relativistic energies. Thus, the present system size may reveal adequate domains through which the pion production mechanism can be investigated fairly well experimentally and theoretically.

\section{INTRODUCTION}

Actually, the synchrophasotron accelerator at Dubna enables equipping beams of $A \geq 1$, in a few $A$ GeV range of energies. This region is a special energy, at which the nuclear limiting fragmentation applies initially [1-7]. The nuclear emulsion is a very useful tool in experimental physics for investigating atomic and nuclear processes. It can be used as a detector of $4 \pi$ space geometry. Moreover, it has a wide range of target mass numbers. In this work $3.7 \mathrm{~A} G \mathrm{GV}{ }^{16} \mathrm{O}$ 
interactions with emulsion nuclei are used. The shower particles are mainly identified in nuclear emulsion as pions. Hence, the pion multiplicity characteristics are investigated according to the emission angular zone, target size, and centrality. The inelastic interaction samples of $3.7 \mathrm{~A} \mathrm{GeV}{ }^{16} \mathrm{O}$ in nuclear emulsion are separated into statistical groups according to the target sizes. Applying the predicted percentages of the Glauber's approach [8], we categorize the data according to the interactions with $\mathrm{H}, \mathrm{CNO}, \mathrm{Em}$, and $\mathrm{AgBr}$ targets separately. The effective mass number of each target group of nuclei is 1 , 14,70 , and 94, respectively. The modified FRITIOF model, MFM, code is used to simulate the present data. It is based on the Lund version 1.6 [9, 10]. The modification was carried out by V. V. Uzhinskii, LIT, JINR, Dubna, Russia, in 1995. The predictions of the MFM are presented in the figures by histograms. The data are approximated in the framework of the multisource thermal model, MSTM [11]. The approximated distributions are presented in the figures by the smooth dashed curves. The characteristics of these curves are obtained as fit parameters.

\section{EXPERIMENTAL DETAILS}

The NIKFI-BR2 nuclear emulsion stack used this experiment is irradiated by ${ }^{16} \mathrm{O}$ beams at the Synchrophasotron of JINR in Dubna, Russia. The beams energy is $3.7 \mathrm{~A} \mathrm{GeV}$. Each emulsion pellicle size is $20 \mathrm{~cm} \times 10 \mathrm{~cm} \times 0.06 \mathrm{~cm}$. Table (1) shows the chemical composition of this emulsion type.

Table 1: Chemical composition of NIKFI-BR2 emulsion.

\begin{tabular}{|c|c|c|c|c|c|c|}
\hline Element & ${ }^{1} \mathrm{H}$ & ${ }^{12} \mathrm{C}$ & ${ }^{14} \mathrm{~N}$ & ${ }^{16} \mathrm{O}$ & ${ }^{80} \mathrm{Br}$ & ${ }^{108} \mathrm{Ag}$ \\
\hline $\begin{array}{c}\text { Atoms } \\
/ \mathbf{c m}^{3} \times 10^{22}\end{array}$ & 3.150 & 1.410 & 0.395 & 0.956 & 1.028 & 1.028 \\
\hline
\end{tabular}

The obeyed methods, equipment, and experimental restrictions are as similar as detailed in experiments [12, 13]. The produced particles are identified in photographic nuclear emulsion, according to the commonly accepted ionization behavior [14, 15], as:

- Shower particles having $\mathrm{g} \leq 1.4 \mathrm{~g}_{\mathrm{p}}$ where $\mathrm{g}$ is the track grain density and $\mathrm{g}_{\mathrm{p}}$ corresponds to the grain density of the minimum ionizing track. These particles are relativistic hadrons, which consist mainly of pions and less than $10 \%$ mesons and baryons. Their multiplicity is denoted as $\mathrm{n}_{\mathrm{s}}$. The notations $n_{s}{ }^{f}$ and $n_{s}{ }^{b}$ correspond to the shower particles emitted in the forward hemisphere, FHS, within $\theta_{\mathrm{lab}}<90^{\circ}$ and in the backward hemisphere, BHS, within $\theta_{\mathrm{lab}}<90^{\circ}$ within $\theta_{\mathrm{lab}}<90^{\circ}$, respectively.

- Grey particles having a range $>3 \mathrm{~mm}$ and $1.4 \mathrm{~g}_{\mathrm{p}}<\mathrm{g} \leq 4.5 \mathrm{~g}_{\mathrm{p}}$; they are mainly recoil protons knocked-out from the target nucleus during the collision. Their kinetic energy ranges from 26 up to $400 \mathrm{MeV}$.

- Black particles having a range $\leq 3 \mathrm{~mm}$ and $\mathrm{g}>4.5 \mathrm{gp}$; they are evaporated target protons with kinetic energy $<26 \mathrm{MeV}$.

- The grey and black particles together amount the group of the target fragments, the so called heavily ionizing particles. These fragments are emitted in the $4 \pi$ space. Their multiplicity is denoted as $N_{h}$.

- The projectile fragments having $Z \geq 1$; they are fragmented nuclei having nearly the same momentum of the incident nucleus. They are emitted in a very narrow forward cone along the direction of incidence.

\section{RESULTS AND DISCUSSION}

\subsection{Interaction Cross-Section}

Since the nuclear emulsion is a homogeneous mixture of different nuclei, the inelastic interactions can be classified into groups according to the target nucleus. In this experiment the events discrimination depends on the theoretical predictions of the Glauber's approach [8]. The present 3.7A GeV ${ }^{16} \mathrm{O}$ inelastic interaction cross section is compared with those due to $60 \mathrm{~A} \mathrm{GeV}{ }^{16} \mathrm{O}$ interacting in FUJI emulsion [16]. The run Glauber's approach code [8] simulates the data which are presented by the histograms in Fig. (1). From the figure, the cross sectional values are nearly the same at the two energies. The Glauber's approach can predict them. The data are approximated by the power law relation of Eq. (1) which is presented by the smooth curves in Fig. (1). The fit parameters, a and b, are listed in Table (2). The fit parameters corresponding to the Glauber's simulations are placed in round brackets. The power is the same within experimental errors, irrespective of the energy. On average, $a=336$ and $b=0.43$. Therefore, Eq. (1) can be rewritten as Eq. (2) which is independent on the energy.

$$
\sigma=a A_{T}^{b}
$$

$\sigma=336 A_{T}^{0.43} \mathbf{m b}$ 


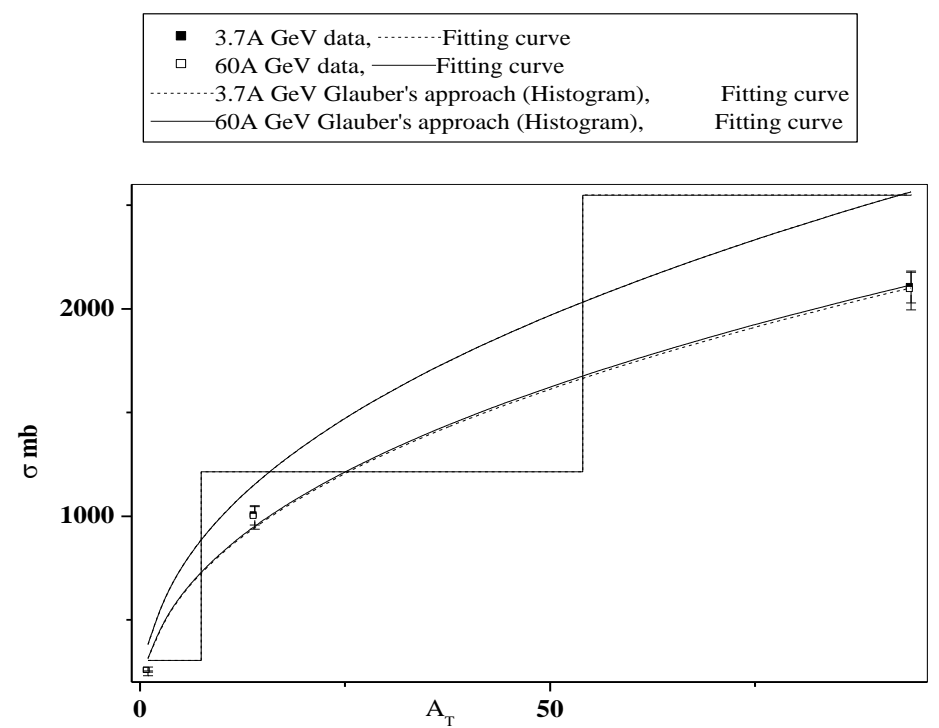

Figure 1: Cross section of ${ }^{16} \mathrm{O}$ inelastic interactions in nuclear emulsion as a function of the target size.

Table 2: Fit Parameters of Eq. (1).

\begin{tabular}{|c|c|c|}
\hline Fit Parameter & a & b \\
\hline$E_{\text {lab }}=3.7 \mathrm{~A} \mathrm{GeV}$ & $314.50 \pm 51.56$ & $(0.42 \pm 0.04$ \\
& $(381.85 \pm 62.73)$ & $0.42 \pm 0.04)$ \\
\hline \multirow{2}{*}{$\mathrm{E}_{\mathrm{lab}}=60 \mathrm{~A} \mathrm{GeV}$} & $312.50 \pm 50.20$ & $(0.42 \pm 0.04)$ \\
\hline
\end{tabular}

\subsection{Backward Emitted Pion Multiplicity Characteristics}

The backward emitted shower particle multiplicity distributions of $3.7 \mathrm{~A} \mathrm{GeV}{ }^{16} \mathrm{O}$ interactions with emulsion nuclei at average impact parameters are shown in Fig. (2).

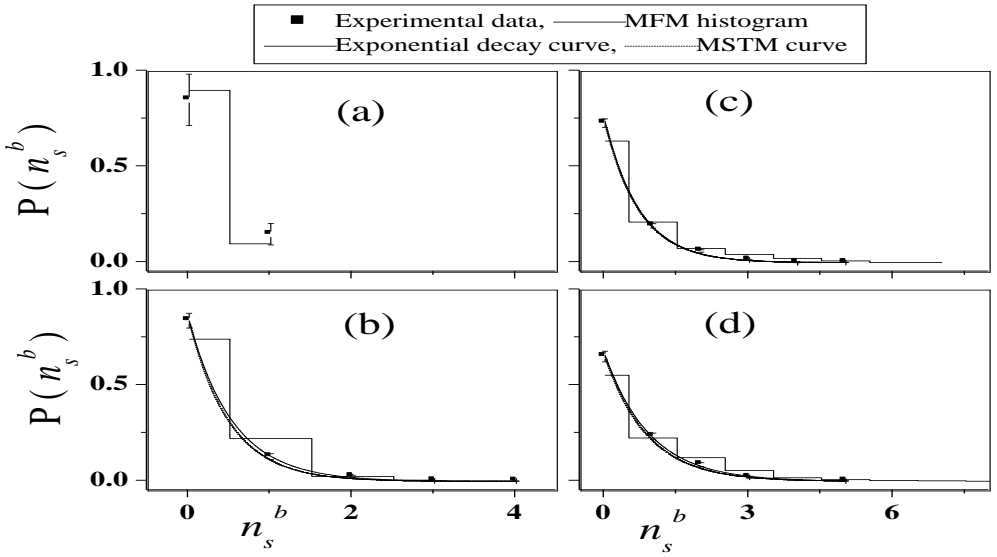

Figure 2: Multiplicity distributions of the backward shower particle emitted in 3.7A GeV ${ }^{16} \mathrm{O}$ interactions with $\mathrm{H}, \mathrm{CNO}$, Em, and $\mathrm{AgBr}$ nuclei. 
From Fig. (2), the characteristic feature of the distribution is the exponential decay shape, irrespective of the target. The multiplicity range (decay tail) exceeds with the target size. The characteristic exponential behavior can be approximated by Eq. (3). The fit parameters, $p_{s}{ }^{b}$ and $\lambda_{s}{ }^{b}$, are listed in Table (3). The data are reproduced well by the MFM. The MSTM approximated curves are superimposed on the exponential decay ones. From Table (3), the average multiplicity, $<n_{s}^{b}>_{\text {Exp }}$, increases with the target size. The predicted average multiplicity, $<n_{s}^{b}>_{\text {MFM }}$, overestimates the experimental one. The overestimation increases with the target size. In the framework of the MSTM the source no, $m_{1}$, is always unity. This implies that a single source is responsible for this particle production system. Hence, the decay shaped curve feature is suggested to indicate a single source production system. The weight factor of the source, $\mathrm{k}_{1}$, increases with the target size. The resulted average multiplicities from the MSTM agree completely with the experimental data.

$P\left(n_{s}^{b}\right)=p_{s}^{b} e^{-\lambda_{s}^{b} n_{s}^{b}}$

Table 3: Characteristic parameters associated with the backward emitted shower particle multiplicity in 3.7A GeV ${ }^{16} \mathrm{O}$ interactions with emulsion nuclei at average impact parameters.

\begin{tabular}{|l|l|l|l|l|l|}
\hline \multicolumn{2}{|l|}{ Target } & $\mathrm{H}$ & $\mathrm{CNO}$ & $\mathrm{Em}$ & $\mathrm{AgBr}$ \\
\hline$p_{s}{ }^{b}$ & - & 0.84 & $0.74 \pm 0.02$ & $0.66 \pm 0.03$ \\
\hline$\lambda_{s}{ }^{b}$ & - & $1.86 \pm 0.02$ & $1.39 \pm 0.04$ & $1.07 \pm 0.04$ \\
\hline \multirow{3}{*}{$\mathrm{j}=1$} & $\mathrm{k}_{1}$ & - & 0.41 & 0.52 & 0.57 \\
\cline { 2 - 6 } & $\mathrm{m}_{1}$ & - & 1 & 1 & 1 \\
\cline { 2 - 6 } & $<\mathrm{n}_{\mathrm{i} 1}>$ & - & 0.49 & 0.72 & 0.88 \\
\hline$<n_{s}^{b}>_{\text {MSTM }}$ & - & 0.20 & 0.37 & 0.50 \\
\hline$<n_{s}{ }^{b}$ Exp $^{<n_{s}{ }^{\text {MFM }}}$ & $0.15 \pm 0.05$ & $0.20 \pm 0.02$ & $0.37 \pm 0.02$ & $0.49 \pm 0.03$ \\
\hline
\end{tabular}

The backward emitted shower particle multiplicity distribution can be determined as a function of the effective target mass number, $A_{T}$, in Fig. (3). The fit parameters are approximated linearly with the target size. The slopes of the lines are -0.009 and -0.002 in insets $a$ and $b$, respectively. In the same respect the intercepts are $2.00 \pm 0.04$ and $0.88 \pm 0.02$.

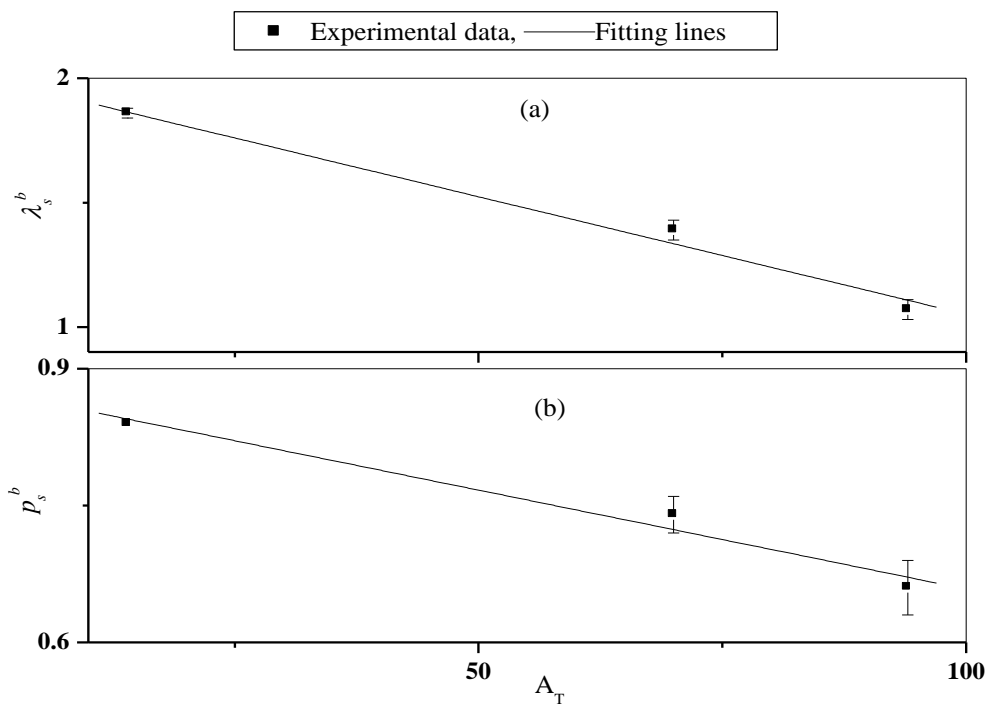

Figure 3: Fit parameters of Eq. (3) as a function of the target mass number.

Independently on the projectile size $\left(A_{\text {proj }}=1\right.$ to 32$)$ or energy $\left(E_{l a b}=2.1 \mathrm{~A}\right.$ to $\left.200 \mathrm{~A} \mathrm{GeV}\right)$, the backward relativistic hadron is produced with probability values of $\sim 20$ to $30 \%$ for interactions with Em target [17]. The values of $<$ $n_{s}^{b}>$ are found to increase with projectile size for $A_{\text {Proj }}<6$. At $A_{\text {Proj }} \geq 6$, they begin to saturate and have a constant value of $\left\langle n_{s}{ }^{b}\right\rangle \sim 0.4$ [17]. The results show also that the energy is not an effective parameter in this backward production [17]. 
Therefore, one can conclude that the backward emitted pion does not come from the fireball nuclear matter or hadronic matter. It is target source particle, regarding the nuclear limiting fragmentation regime.

\subsection{Forward Emitted Pion Multiplicity Characteristics}

The forward emitted shower particle multiplicity distributions of $3.7 \mathrm{~A} \mathrm{GeV}{ }^{16} \mathrm{O}$ interactions with emulsion nuclei at average impact parameters are shown in Fig. (4). The interactions with $\mathrm{H}, \mathrm{CNO}, \mathrm{Em}$, and $\mathrm{AgBr}$ are presented in insets a, b, c, and d, respectively.
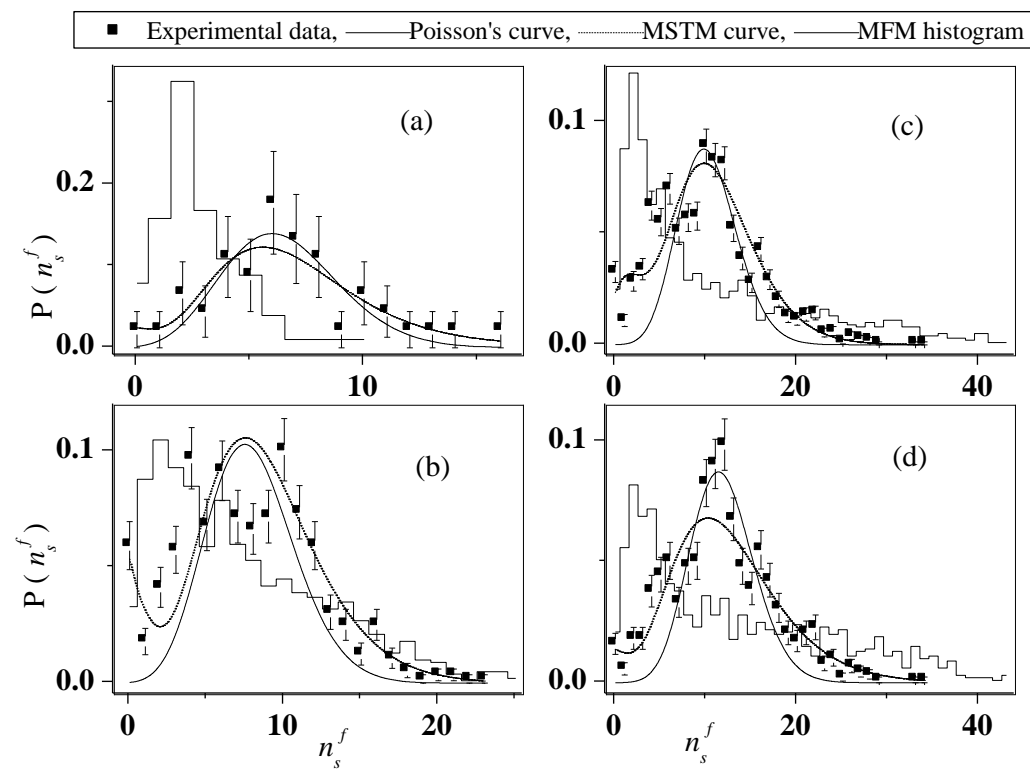

Figure 4: Multiplicity distributions of the forward emitted shower particle in $3.7 \mathrm{~A} \mathrm{GeV}{ }^{16} \mathrm{O}$ interactions with $\mathrm{H}$, $\mathrm{CNO}, \mathrm{Em}$, and $\mathrm{AgBr}$ nuclei at average impact parameters.

Unlike the observed behavior of the backward emitted shower particle, the characteristic feature, here, is the peaking curve shapes. The multiplicity range as well as the broadening of the distributions increases with target size. The geometrical model considering the overlap size between target and projectile seems to be effective in drawing the characteristic features of the distributions. Accordingly the effect of the target size is reflected on the impact parameter value and consequently on the energy participation, which is the main effective parameter in particle creation. In this concept, the average multiplicity increases with the target size as shown in Table (4). The MFM can not estimate the data. It reproduces qualitatively the distributions at higher multiplicities only in insets $\mathrm{b}, \mathrm{c}$, and $\mathrm{d}$. The average multiplicities, $<n_{s}{ }^{{ }}$ $>_{\text {MFM }}$, can be predicted by the MFM except for $\mathrm{H}$ target nuclei events. The distributions are fitted by the Poisson's law of Eq. (4).

$P\left(n_{s}^{f}\right)=p_{s}^{f} \frac{<n_{s}^{f}>^{n_{s}^{f}}}{n_{s}^{f} !} e^{-<n_{s}^{f}>}$

The normalization factor, $p_{s}^{f}$, and $<n_{s}^{f}>$ Poisson of Eq. (4) are obtained as fit parameters. They are placed in Table (4). The average multiplicity is predicted well by Poisson's law. The dispersion of the forward emitted shower particle multiplicity is defined by Eq. (5). The experimental dispersion values, $D_{\text {Exp }}$, and the simulated values, $D_{\mathrm{MFM}}$, are listed in Table (4). From the table the dispersion increases with the target size. The simulated values overestimate the experimental ones except for the events associated with $\mathrm{H}$ target nuclei.

$D=\sqrt{<\left(n_{s}^{f}\right)^{2}>-<n_{s}^{f}>^{2}}$ 
Table 4: Characteristic parameters associated with the forward emitted shower particle multiplicity in 3.7A GeV ${ }^{16} \mathrm{O}$ interactions with emulsion nuclei at average impact parameters.

\begin{tabular}{|c|c|c|c|c|c|}
\hline \multicolumn{2}{|l|}{ Target } & $\mathrm{H}$ & $\mathrm{CNO}$ & Em & $\mathrm{AgBr}$ \\
\hline \multicolumn{2}{|l|}{$p_{s}^{\prime}$} & $0.89 \pm 0.08$ & $0.73 \pm 0.08$ & $0.70 \pm 0.06$ & $0.75 \pm 0.05$ \\
\hline \multicolumn{2}{|l|}{$<n_{s}{ }^{\prime}>_{\text {Poisson }}$} & $6.45 \pm 0.33$ & $7.95 \pm 0.44$ & $10.22 \pm 0.40$ & $11.83 \pm 0.35$ \\
\hline \multicolumn{2}{|l|}{$<n_{s}^{f}>\operatorname{Exp}$} & $6.64 \pm 1.08$ & $7.65 \pm 0.32$ & $10.10 \pm 0.26$ & $11.84 \pm 0.40$ \\
\hline \multicolumn{2}{|l|}{$<n_{s}^{\prime}>_{\mathrm{MFM}}$} & 2.60 & 7.18 & 10.15 & 12.86 \\
\hline \multicolumn{2}{|l|}{$D_{s}^{\prime} \operatorname{Exp}$} & $3.39 \pm 0.55$ & $4.43 \pm 0.19$ & $5.60 \pm 0.16$ & $5.70 \pm 0.19$ \\
\hline \multicolumn{2}{|l|}{$D_{s}^{f} \mathrm{MFM}$} & 1.84 & 5.41 & 9.66 & 10.54 \\
\hline \multirow[t]{3}{*}{$\mathrm{j}=1$} & $\mathrm{k}_{1}$ & 0.80 & 0.90 & 0.80 & 0.86 \\
\hline & $\mathrm{m}_{1}$ & 5 & 6 & 7 & 5 \\
\hline & $<n_{i 1}>$ & 1.41 & 1.50 & 1.67 & 2.58 \\
\hline \multirow[t]{3}{*}{$\mathrm{j}=2$} & $\mathrm{k}_{2}$ & 0.20 & 0.05 & 0.12 & 0.04 \\
\hline & $m_{2}$ & 1 & 2 & 2 & 2 \\
\hline & $<\mathrm{n}_{\mathrm{i} 2}>$ & 8.256 & 1.421 & 2.402 & 0.002 \\
\hline \multirow[t]{3}{*}{$\mathrm{j}=3$} & $\mathrm{k}_{3}$ & - & 0.05 & 0.08 & 0.10 \\
\hline & $m_{3}$ & - & 1 & 1 & 1 \\
\hline & $<n_{i 3}>$ & - & 0.93 & 3.41 & 7.28 \\
\hline \multicolumn{2}{|l|}{$\left.<n_{s}^{\prime}\right\rangle_{\text {MSTM }}$} & 7.29 & 8.29 & 10.20 & 11.82 \\
\hline
\end{tabular}

In the framework of the MSTM, the distributions are reproduced well. The obtained average multiplicities, $<n_{s}^{f}$ >MSTM, agree perfectly with the experimental data. Table (4) data indicate that there are 3 subgroups of sources responsible for this production system. However, the $3^{\text {rd }}$ subgroup has weak weight factors. In $\mathrm{H}$ target data 2 subgroups are found only. The $1^{\text {st }}$ subgroup forms, at least, $80 \%$ of the sources. It consists of 5 to 7 sources. Hence, the forward emitted pion multiplicity distribution is a superposition of multisource characterized by the peaking shaped curve. It is reasonable to say that the mechanism of particle production in the FHS is completely different from that in the BHS.

\subsection{Production Probability of Pion in BHS}

The percentage probability of the backward emitted shower particle production, $\mathrm{P}\left(n_{s}^{b}>0\right) \%$, is defined as, the number of events having $\left(n_{s}^{b}>0\right)$ normalized to the total sample of events. In Fig. (5) this probability is evaluated as a function of the target mass number for the present interactions.

From Fig. (5), one observes the strong dependence of backward relativistic hadron production on the target size. This strong dependence is evaluated linearly by Eq. (6) and presented in Fig. (5) by the straight lines. Independently on the projectile size $\left(A_{\text {Proj }}=1\right.$ to 32$)$ or energy $\left(E_{l a b}=2.1 A\right.$ to $\left.200 \mathrm{~A} \mathrm{GeV}\right)$, the backward relativistic hadron is produced with probability values of $\sim 20$ to $30 \%$ for interactions with Em target [17]. The theoretical predictions of the FRITIOF model agree with the data, especially at 3.7A GeV. The fit parameters $\mu$ and $v$ are listed in Table (6).

$P\left(n_{s}^{b}>0\right) \%=\mu+v A_{T}$ 


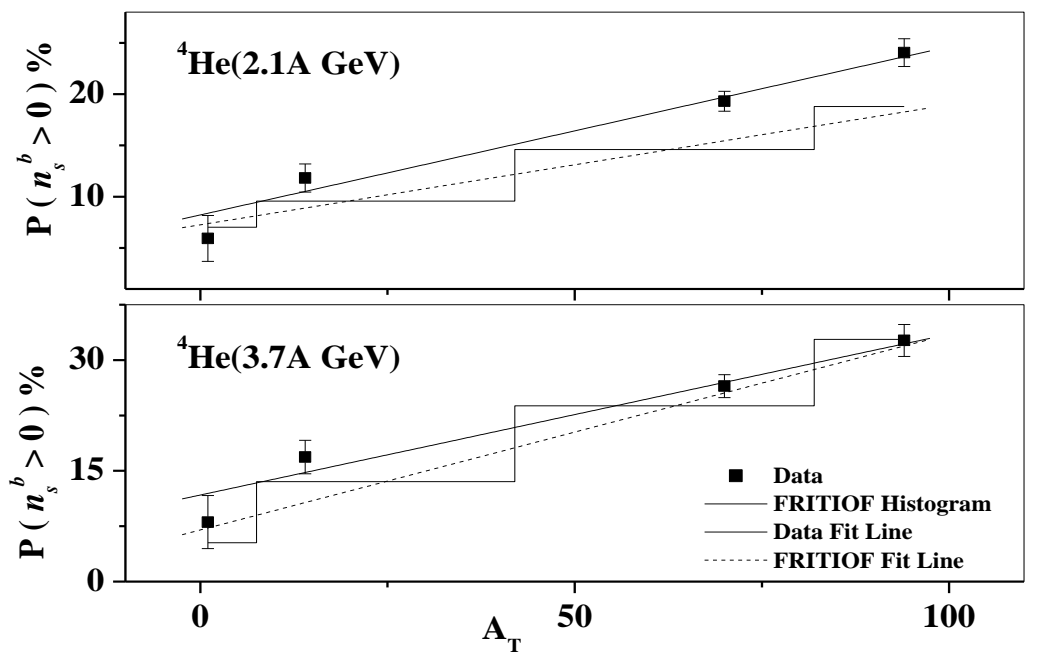

Figure 5: The probability of the backward emitted shower particle multiplicity, in the interactions of 2.1A and 3.7A

$\mathrm{GeV}^{4} \mathrm{He}$ with emulsion nuclei, as a function of the target mass number, together with the predictions of the modified FRITIOF model and the fitting lines.

Table 6: The fit parameters of Eq. (6).

\begin{tabular}{|c|c|c|}
\hline$E_{\text {lab }} / \mathrm{A} \mathrm{GeV}$ & $\mu$ & $v$ \\
\hline \multirow{2}{*}{2.1} & $8.22 \pm 1.42$ & $0.16 \pm 0.02$ \\
& $(7.26 \pm 0.69)$ & $(0.12 \pm 0.01)$ \\
\hline \multirow{2}{*}{3.7} & $11.72 \pm 2.22$ & $0.22 \pm 0.03$ \\
& $(6.99 \pm 2.16)$ & $(0.27 \pm 0.04)$ \\
\hline
\end{tabular}

\subsection{Average Multiplicity}

The average multiplicities of the forward and backward shower particles, emitted in the present interactions, are correlated with the target size in Fig. (6). The correlation reveals a linear dependence, for the backward emitted shower particle, presented by the straight lines. Eq. (7) approximates the values of fit parameters, $a_{s}{ }^{b}$ and $b_{s}{ }^{b}$, to be listed in Table (7). The model reproduces the linear correlation especially at 3.7A GeV. The slope parameter $\sim 0$ and the intercept parameter $\sim 0.1$, irrespective of the energy.

$<n_{s}^{b}>=a_{s}^{b}+b_{s}^{b} A_{T}$ 

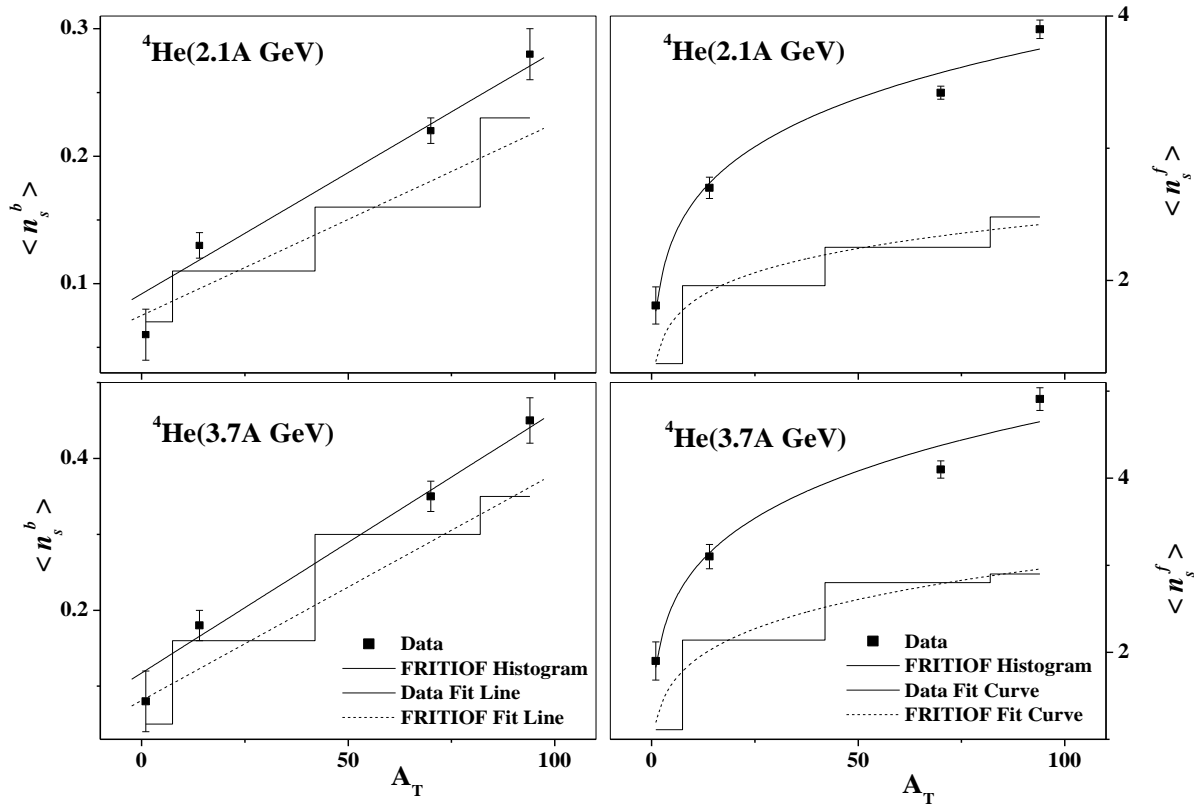

\section{Figure 6: The dependence correlations of the forward and backward emitted shower particle average} multiplicities on the target size in the present interactions, together with the theoretical prediction.

In experiment [17], using a wide range of projectile size ( $A_{\text {Proj }}=1$ to 32 ) interacting in nuclear emulsion at Dubna energy, the values of $\left\langle n_{s}^{b}\right\rangle$ are found to increase with projectile size for $A_{\text {Proj }}<6$. At $A_{\text {Proj }} \geq 6$, they began to saturate and had a constant value of $\left\langle n_{s}^{b}\right\rangle \sim 0.4$. In this experiment the results imply that the energy is not an effective parameter in backward shower emission. Therefore, one can conclude that, while the average shower particle multiplicity, emitted in the BHS, depends on the target size, it depends neither on the projectile size nor energy. This confirms our expectation that, the backward relativistic hadron does not come from the fireball nuclear matter or hadronic matter. They are target source particles, regarding the nuclear limiting fragmentationregime. In Fig. (6), the forward emitted shower particle average multiplicity shows higher values than the backward ones. It increases with the energy as well as target size. The increase with the target size, here, does not mean that this particle is a target source but the target size enhances in the total system size which affects the participant matter size. Although the dependence on the target size is strong, however it has often a tendency of saturation at $A_{T}>14$. This behavior may be reflected on the dependence which is approximated well by a power law relation of Eq. (8). This approximation is presented in Fig. (6) by the smooth curves. The fit parameters, $a_{s}{ }^{f}$ and $b_{s}{ }^{f}$, are listed in Table (7). From Fig. (6), one can observe also that the model underestimates the data. Abdelsalam et al [17] determine the dependence of $\left\langle n_{s}\right\rangle$ on the projectile mass number at Dubna energy. They find that dependence as; $<n_{s}^{f}>=1.89 A_{\text {proj }}^{0.56}$, i. e. $<n_{s}^{f}>\propto r_{\text {proj }}^{2 / 3}$

$<n_{s}^{f}>=a_{s}^{f} A_{T}^{b_{s}^{f}}$

Comparing this dependence by Eq. (8), it can be observed that the majority contribution of the participant matter is accounted from the projectile throughout the production of the forward emitted shower particle. Thus, while the production source of the backward emitted shower particle is the target fragmentation system the forward emitted one is originated mainly from a creation system provided by the participant energy.

Table 7: The fit parameters of Eq. (7) and Eq. (8).

\begin{tabular}{|c|c|c|c|c|}
\hline $\mathrm{E}_{\mathrm{lab}} / \mathrm{A} \mathrm{GeV}$ & $a_{s}{ }^{b}$ & $b_{s}{ }^{b}$ & $a_{s}{ }^{f}$ & $b_{s}{ }^{\prime}$ \\
\hline \multirow{2}{*}{2.1} & $0.092 \pm 0.016$ & $0.002 \pm 0$ & $1.77 \pm 0.13$ & $0.17 \pm 0.02$ \\
& $(0.075 \pm 0.016)$ & $(0.002 \pm 0)$ & $(1.39 \pm 0.07)$ & $(0.12 \pm 0.01)$ \\
\hline \multirow{2}{*}{3.7} & $0.117 \pm 0.020$ & $0.003 \pm 0$ & $1.83 \pm 0.23$ & $0.21 \pm 0.03$ \\
& $(0.081 \pm 0.029)$ & $(0.003 \pm 0)$ & $(1.20 \pm 0.09)$ & $(0.20 \pm 0.02)$ \\
\hline
\end{tabular}




\section{CONCLUSIONS}

From the analysis of $3.7 \mathrm{~A} \mathrm{GeV}{ }^{16} \mathrm{O}-$ nucleus interactions, using photographic nuclear emulsion, we conclude the following:

1- The inelastic interaction cross section of ${ }^{16} \mathrm{O}$ in nuclear emulsion is approximated as a function of the target mass number. In the present energy region, the cross section is independent on the energy. It can be determined in the light of the Glauber's multiple scattering theory.

2- The dominant mechanism characterizing the backward shower particle production is the decay behavior. There is no energy effect on the backward production. The multiplicity distribution of this hadron is expressed in terms of the target size. While the production probability of this hadron is independent on the projectile size or energy it increases linearly with the target size. While the average backward shower particle multiplicity tends to a limited value $\sim 0.4$, irrespective of the projectile size or energy, it increases linearly with the target size. Hence, the main effective parameter is the target size, regarding the nuclear limiting fragmentation beyond $1 \mathrm{~A} \mathrm{GeV}$. Thus, such hadron is expected to be decayed through the de-excitation of the excited target nucleus as similar as the compound nucleus mechanism.

3- In the FHS the shower particle multiplicity distributions are peaking shaped, where they can be described well by the Gaussian shapes. The production of the forward emitted relativistic hadron is attributed to a mechanism, which is completely different from that in BHS. Although the target nucleus is not the source of the forward relativistic hadron, however the target size is an effective parameter in this production as well as the projectile size. The geometrical concept underlying the nuclear fireball model may interpret the effect of the projectile and target sizes in particle production at high energy. The effect of the target size on the forward shower particle production is reflected on their multiplicity characteristics at each target. Regarding the incident energy role as a principal parameter affecting the forward relativistic hadron production, this system of production is regarded as a particle creation system, in which the particles are sourced from hadronic matter or fireball nuclear matter.

4- The modified FRITIOF model can predict the system of the relativistic hadron production in the BHS well. In the FHS the hadronization system can be described satisfactorily. This suggests that the Reggeon picture can be considered as a plausible development to the Model. Sometimes underestimations or overestimations are observed in the model predictions with experimental data. This may require a modern approach in describing nuclear cascading.

5- The decay shape indicates a single source production system and the peaking one is a multisource superposition. This is confirmed well in the by the multisource thermal model predictions.

\section{ACKNOWLEDGEMENT}

We owe much to Vekseler and Baldin High Energy Laboratory, JINR, Dubna, Russia, for supplying us the photographic emulsion plates irradiated at Synchrophasotron.

\section{REFERENCES}

1. Benecke J, Chou T T, Yang C N, Yen E. Phys. Rev.,1969, 188, 2159.

2. Fu-Hu Liu. Chinese Journal of Physics,2002, 40, 159.

3. Ahmad M S, Khan M Q R, Hasan R. Nucl. Phys. A, 1989, 499, 821.

4. Webber W R. Proceedings of the international Cosmic Ray Conference, Vol. 8, P. 65, Moscow, USSR (1987).

5. Lindstorm P L, Greiner D E, Heckman H H, Cork B. Lawrence Berkeley Laboratory Report, LBL-3650, 1975.

6. Olson D L, Berman B L, Grenier D E, Heckman H H, Lindstrom P J, Crawford H J. Phys. Rev. C, 1983, $28,1602$.

7. El-Nagdy M S, Abdelsalam A, Abou-Moussa Z, Badawy B M. Can. J. Phys.,2013, 91, 737.

8. Shmakov S Yu, Uzhinskii V V. Com. Phys. Comm.,1989, 54, 125.

9. Andersson B, Gustafson G, Nilsson-Almqvist B. Nucl. Phys. B,1987, 281, 289.

10. Nilsson-Almqvist B, Stenlund E. Comp. Phys. Comm.,1987, 43, 387.

11. Fu-Hu Liu Nucl. Phys. A, 2008, 810, 159.

12. Abdelsalam A, Metwalli N, Kamel S, Aboullela M, Badawy B M, Abdallah N. Can. J. Phys.,2013, 91, 438.

13. Abdelsalam A, Badawy B M, Hafiz M. J. Phys. G: Nucl. Part. Phys.,2012, 39, 105104.

14. Powell C F, Fowler F H, Perkins D H. The Study of Elementary Particles by the Photographic Method, Pergamon Press. London; New York, Paris, Los Angles, 474 (1958).

15. Barkas H. Nuclear Research Emulsion, Vol. I, Technique and Theory Academic Press Inc., (1963).

16. Abdelsalam A., El-Nagdy M. S., Badawy B. M., Osman W., Fayed M. International Journal of Modern Physics E, 2016, 25, 1650034.

17. Abdelsalam A, El-Nagdy M S, Badawy B M; Can. J. Phys.,2011, 89, 261. 